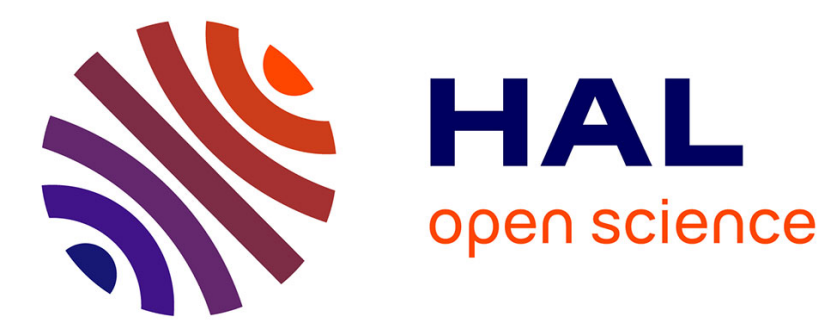

\title{
On-sky demonstration of matched filters for wavefront measurements using ELT-scale elongated laser guide stars
}

\author{
A. G. Basden, L. Bardou, D. Bonaccini Calia, T. Buey, M. Centrone, F. \\ Chemla, J. L. Gach, E. Gendron, D. Gratadour, I. Guidolin, et al.
}

\section{To cite this version:}

A. G. Basden, L. Bardou, D. Bonaccini Calia, T. Buey, M. Centrone, et al.. On-sky demonstration of matched filters for wavefront measurements using ELT-scale elongated laser guide stars. Monthly Notices of the Royal Astronomical Society, 2017, 466 (4), pp.5003-5010. 10.1093/mnras/stx062 . hal-01678425

\section{HAL Id: hal-01678425 \\ https://hal.science/hal-01678425}

Submitted on 15 May 2018

HAL is a multi-disciplinary open access archive for the deposit and dissemination of scientific research documents, whether they are published or not. The documents may come from teaching and research institutions in France or abroad, or from public or private research centers.
L'archive ouverte pluridisciplinaire HAL, est destinée au dépôt et à la diffusion de documents scientifiques de niveau recherche, publiés ou non, émanant des établissements d'enseignement et de recherche français ou étrangers, des laboratoires publics ou privés. 


\section{Durham Research Online}

\section{Deposited in DRO:}

16 March 2017

\section{Version of attached file:}

Published Version

\section{Peer-review status of attached file:}

Peer-reviewed

\section{Citation for published item:}

Basden, A. G. and Bardou, L. and Bonaccini-Calia, D. and Buey, T. and Centrone, M. and Chemla, F. and Gach, J. L. and Gendron, E. and Gratadour, D. and Guidolin, I. and Jenkins, D. R. and Marchetti, E. and Morris, T. J. and Myers, R. M. and Osborn, J. and Reeves, A. P. and Reyes, M. and Rousset, G. and Stangalini, M. and Townson, M. J. and Vidal, F. (2017) 'On-sky demonstration of matched filters for wavefront measurements using ELT-scale elongated laser guide stars.', Monthly notices of the Royal Astronomical Society., 466 (4). pp. 5003-5010.

\section{Further information on publisher's website:}

https://doi.org/10.1093/mnras/stx062

\section{Publisher's copyright statement:}

This article has been accepted for publication in Monthly notices of the Royal Astronomical Society (C): 2017 The Authors Published by Oxford University Press on behalf of the Royal Astronomical Society. All rights reserved.

Additional information:

Use policy

The full-text may be used and/or reproduced, and given to third parties in any format or medium, without prior permission or charge, for personal research or study, educational, or not-for-profit purposes provided that:

- a full bibliographic reference is made to the original source

- a link is made to the metadata record in DRO

- the full-text is not changed in any way

The full-text must not be sold in any format or medium without the formal permission of the copyright holders.

Please consult the full DRO policy for further details. 


\title{
On-sky demonstration of matched filters for wavefront measurements using ELT-scale elongated laser guide stars
}

\author{
A. G. Basden, ${ }^{1 \star}$ L. Bardou, ${ }^{2}$ D. Bonaccini Calia, ${ }^{3}$ T. Buey, ${ }^{2}$ M. Centrone, ${ }^{4}$ F. Chemla, ${ }^{5}$ \\ J. L. Gach, ${ }^{6}$ E. Gendron, ${ }^{2}$ D. Gratadour, ${ }^{2}$ I. Guidolin, ${ }^{3}$ D. R. Jenkins, ${ }^{1}$ E. Marchetti, ${ }^{3}$ \\ T. J. Morris, ${ }^{1}$ R. M. Myers,${ }^{1}$ J. Osborn, ${ }^{1}$ A. P. Reeves, ${ }^{1}$ M. Reyes, ${ }^{7}$ G. Rousset,${ }^{2}$ \\ G. Lombardi, ${ }^{8}$ M. J. Townson ${ }^{1}$ and F. Vidal $^{2}$ \\ ${ }^{1}$ Department of Physics, Durham University, South Road, Durham DH1 3LE, UK \\ ${ }^{2}$ LESIA, Observatoire de Paris - Paris Sciences et Lettres, CNRS, Université P. et M. Curie - Sorbonne Universités, Université Paris Diderot - Sorbonne Paris \\ Cité, 5, Place Jules Janssen, F-92190 Meudon, France \\ ${ }^{3}$ European Southern Observatory, D-85748 Garching, Germany \\ ${ }^{4}$ INAF-OAR National Institute for Astrophysics, Via Frascati 33, I-00078 Monte Porzio Catone (RM), Italy \\ ${ }^{5}$ GEPI, Observatoire de Paris - Paris Sciences et Lettres, CNRS, Université Paris Diderot - Sorbonne Paris Cité, 5, Place Jules Janssen, \\ F-92190 Meudon, France \\ ${ }^{6}$ Laboratoire d'Astrophysique de Marseille, 38 rue F. Joliot-Curie, F-13388 Marseille Cedex 13, France \\ ${ }^{7}$ Instituto de Astrofisica de Canarias, Via Lactea, E-38205 La Laguna, Tenerife, Spain \\ ${ }^{8}$ Gran Telescopio Canarias, Cuesta de San José, s/n - E-38712, Breña Baja, La Palma, Spain
}

Accepted 2017 January 9. Received 2016 November 29; in original form 2016 November 29

\begin{abstract}
The performance of adaptive optics systems is partially dependent on the algorithms used within the real-time control system to compute wavefront slope measurements. We demonstrate the use of a matched filter algorithm for the processing of elongated laser guide star (LGS) Shack-Hartmann images, using the CANARY adaptive optics instrument on the $4.2 \mathrm{~m}$ William Herschel Telescope and the European Southern Observatory Wendelstein LGS Unit placed $40 \mathrm{~m}$ away. This algorithm has been selected for use with the forthcoming Thirty Meter Telescope, but until now had not been demonstrated on-sky. From the results of a first observing run, we show that the use of matched filtering improves our adaptive optics system performance, with increases in on-sky $H$-band Strehl measured up to about a factor of 1.1 with respect to a conventional centre of gravity approach. We describe the algorithm used, and the methods that we implemented to enable on-sky demonstration.
\end{abstract}

Key words: instrumentation: adaptive optics - methods: numerical.

\section{INTRODUCTION}

Adaptive optics (AO) systems have reached a new level of maturity in recent years, with many new strategies and algorithms being demonstrated and realized in facility class systems, e.g. eXtreme AO (Fusco et al. 2006; Macintosh et al. 2012; Jovanovic et al. 2015) and multiconjugate adaptive optics (Rigaut et al. 2012). Future systems for the forthcoming Extremely Large Telescopes (ELTs) are currently under development, and it is important that optimum performance is obtained for these expensive facilities. A key area of investigation is optimization of algorithms that are used in the AO real-time pipeline, to extract maximum information from the photons received by the wavefront sensors (WFSs), and using a priori knowledge of the atmosphere to compute the best match of

${ }^{\star}$ E-mail: a.g.basden@durham.ac.uk deformable mirror (DM) surface to the phase perturbations introduced by atmospheric turbulence.

In order to increase sky coverage of AO-corrected observations (which are limited by the availability of bright natural guide stars), laser guide stars (LGSs) are used (Foy \& Labeyrie 1985). Artificial stars can be created in the mesosphere, at about $90 \mathrm{~km}$ above the surface of the Earth, using a laser tuned to the resonance of sodium atoms. However, due to the thickness of the sodium layer, this leads to an elongation of the spots in a Shack-Hartmann WFS when sub-apertures view the LGS off-axis, due to perspective (Gilles \& Ellerbroek 2006). This elongation is approximately proportional to the distance between the sub-aperture (projected onto the telescope pupil) and the laser launch location (projected into the plane of the primary mirror). For the European ELT (E-ELT), this distance can reach about $40 \mathrm{~m}$ (Tamai \& Spyromilio 2014, for a small number of most affected sub-apertures), and so spots extend for up to 20-30 arcsec along the elongation direction, depending on the 
precise distribution of mesospheric sodium atoms. Spots that are this large introduce several challenges for wavefront sensing: flux is spread over a larger number of pixels and so signal-to-noise ratio is reduced, and sensitivity to spot motion along and perpendicular to the elongation direction differs.

Here, we consider the use of a noise-optimal matched filter algorithm (Gilles \& Ellerbroek 2006, 2008; Conan et al. 2009) for calculation of local wavefront gradients in a Shack-Hartmann WFS. We report on successful on-sky operation of this algorithm, taking advantage of a dedicated experiment set to evaluate the performance of AO using ELT-scale elongated LGSs (Rousset et al. 2014). This experiment is based on the use of the CANARY AO technology demonstrator instrument (Myers et al. 2008; Gendron et al. 2011) at the $4.2 \mathrm{~m}$ William Herschel Telescope (WHT) coupled to the European Southern Observatory (ESO) Wendelstein LGS Unit (WLGSU Bonaccini Calia et al. 2012) with the laser being launched $40 \mathrm{~m}$ away from the WHT. Performance of the AO system when using the matched filter algorithm is compared with that obtained using a well-optimized centre of gravity (COG) approach (see Section 3.3.2 for further details about the optimization). The matched filter algorithm has been selected for use with the forthcoming Thirty Meter Telescope (TMT; Nelson \& Sanders 2008), and, as we show, also has relevance for other telescopes. Previous authors have also compared the performance of different algorithms used for wavefront slope determination for Shack-Hartmann WFSs (Thomas et al. 2006; Basden et al. 2015). Here, we provide results of the first on-sky verification of one of these.

The CANARY AO system has seen several phases of operation using many different WFSs and DMs (Basden et al. 2016). Here, we operate in phase D mode (Rousset et al. 2014), with a single on-axis sodium LGS, an on-axis natural guide star (NGS) truth WFS and, optionally, three off-axis NGSs (which we do not consider further here). Phase D of CANARY is designed to emulate a sub-pupil of the E-ELT, with the LGSs being launched far off-axis so that significant spot elongation effects can be studied. The WFSs all have $7 \times 7$ sub-apertures, of which 36 are valid (not significantly vignetted). The LGS WFS has $30 \times 30$ pixels per sub-aperture, and is based on an electron multiplying CCD (EMCCD) detector with sub-electron readout noise (Gach et al. 2016). It is equipped with a dedicated fast steering mirror to stabilize the LGS spots in the sub-apertures. Wavefront correction is performed using an $8 \times 8$ actuator DM with 52 active actuators and a dedicated tip/tilt mirror.

We concentrate our efforts on matched filtering of highly elongated sodium LGS spots, as shown in Fig. 1, though we have also tested with NGS spot patterns. However, on-sky observing time was limited and so we do not present results for NGSs: we did not take comprehensive NGS measurements once the AO loop was successfully closed.

The matched filter algorithm has been developed to help mitigate some of the problems introduced by guide star elongation, including reduction in flux per pixel and sensitivity loss due to structure. Previous studies, e.g. Gilles \& Ellerbroek (2006) have found that spot position estimation error is significantly impacted by this elongation, and that using a matched filter can reduce this effect, particularly in the presence of low photon signal and high detector readout noise.

Unfortunately, the matched filter algorithm is non-linear, i.e. estimated position and hence root-mean-square (RMS) error depends non-linearly on distance of the spot from the reference position of the matched filter. However, a linearity extension has been proposed (Gilles \& Ellerbroek 2008) that we use to extend the linearity of this algorithm up to about 1 pixel of displacement. Additionally, because

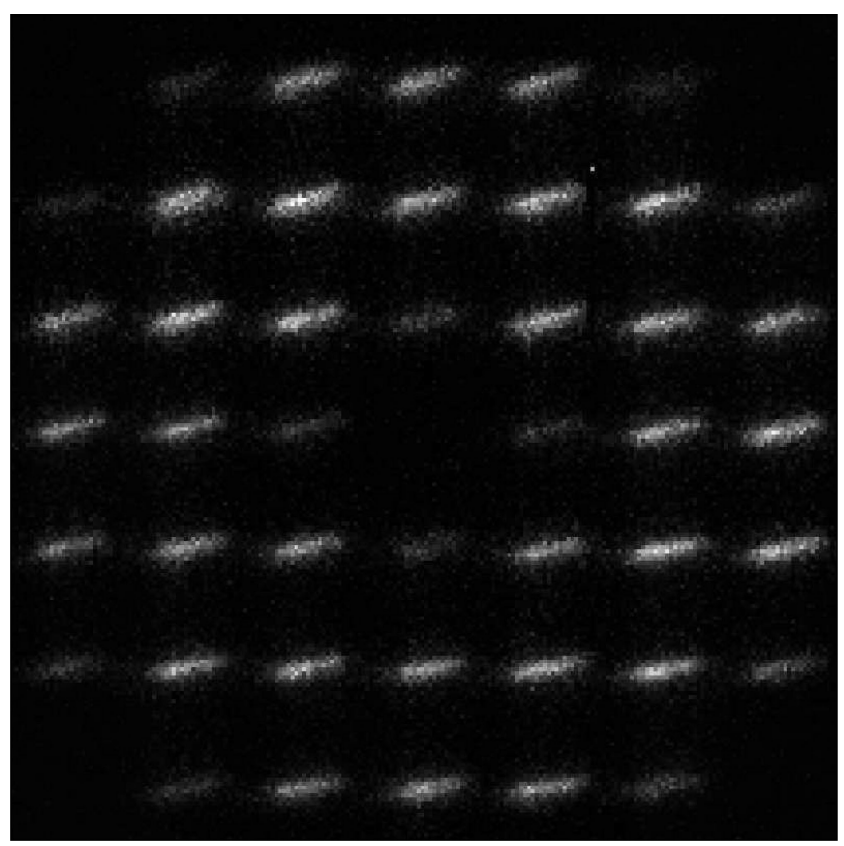

Figure 1. A figure showing a single WFS frame of elongated LGS spots recorded by the CANARY AO system on 2016 September 20. This image has $30 \times 30$ pixels per sub-aperture, and a pixel scale of 0.65 arcsec per pixel.

we operate in closed loop (as with the TMT), the AO system helps to maintain the spots in a central position, though we note that spot motion can still be large for wide-field LGS systems and ground layer AO (GLAO) systems. The pixel scale of the WFS that we use is also fairly large, at 0.65 arcsec per pixel, principally so that a reasonable sub-aperture size can have sufficient field of view to image the entire LGS spot, and therefore, under reasonable seeing conditions (e.g. 0.7 arcsec), LGS spot motion is small, within the linear range of the matched filter algorithm.

In the case of the forthcoming E-ELT, LGSs are launched from the edge of the telescope pupil, and so the degree of elongation varies across a Shack-Hartmann WFS from close to zero (for subapertures near the launch location) to about $40 \mathrm{~m}$ (for sub-apertures furthest from the launch location), which corresponds to tens of arcseconds of angular diameter for typical sodium layer profiles and thickness. In this case, a COG algorithm can be expected to perform well for sub-apertures with little elongation, and poorly for well-elongated sub-apertures. The matched filter algorithm is able to improve measurement accuracy for all sub-apertures, with the largest gain being for those that are most elongated.

In Section 2, we describe the technique that we use to build and apply the matched filters. In Section 3, we present on-sky results, and we conclude in Section 4.

\section{MATCHED FILTERS FOR WAVEFRONT GRADIENT ESTIMATION}

We follow the derivation of the matched filter as given by Gilles \& Ellerbroek (2006), with an extension for dynamic range (Gilles \& Ellerbroek 2008). This is outlined as follows.

The sub-aperture pixel intensity is modelled as

$\boldsymbol{I}_{m}(\boldsymbol{x})=\boldsymbol{I}_{0}(\boldsymbol{x})+\mathbf{G} \cdot \boldsymbol{x}+\boldsymbol{\eta}$, 
where $I_{m}$ is the intensity of a pixel at location $\boldsymbol{x}, I_{0}$ is the high light level on-axis intensity (assumed to be noiseless), $G$ is the derivative of $I_{0}$ in the $x$ and $y$ directions (the pixel gain, or Jacobian) having components $G_{x}$ and $G_{y}$, and $\eta$ is the measurement noise vector due to the photon shot noise and detector readout noise.

The minimization of the difference between measured pixel intensity and model pixel intensity (equation 1) is required to compute the estimate of the spot location, and the derivation is given by Gilles $\&$ Ellerbroek (2008). In summary (with small modifications made by us to allow for variations in flux measurements), estimated spot position is given by

$\boldsymbol{s}_{x}=\frac{\mathbf{R} \cdot \boldsymbol{I}}{\sum \boldsymbol{I}}$

where $\boldsymbol{s}_{x}$ is the desired slope measurement, $I$ is the sub-aperture image (flattened into a one-dimensional array) and $\sum I$ is a measure of the total flux within I. $R$ (the matched filter), which has both $x$ and $y$ components, is given by

$\boldsymbol{R}=f \boldsymbol{M}\left(\boldsymbol{H}^{T} \boldsymbol{C}^{-1} \boldsymbol{H}\right)^{-1} \boldsymbol{H}^{T} \boldsymbol{C}^{-1}$

with $\mathbf{C}$ being the noise covariance matrix (a diagonal matrix of side length equal to the number of pixels within a sub-aperture), with entries equal to $I_{0}+\sigma^{2}$ for photon and detector noise where $\sigma$ is the detector readout noise, $f$ is the sum of flux in $I_{0}$,

$\mathbf{M}=\left[\begin{array}{ccccccc}1 & 0 & 0 & 1 & -1 & 0 & 0 \\ 0 & 1 & 0 & 0 & 0 & 1 & -1\end{array}\right]$

and

$\mathbf{H}=\left[\begin{array}{lllllll}\boldsymbol{G}_{x} & \boldsymbol{G}_{y} & \boldsymbol{I}_{0} & \boldsymbol{I}_{1} & \boldsymbol{I}_{2} & \boldsymbol{I}_{3} & \boldsymbol{I}_{4}\end{array}\right]$.

Here, $I_{1,2,3,4}$ are the high light level intensities of the spot, shifted in the $+\mathrm{x},-\mathrm{x},+\mathrm{y}$ and $-\mathrm{y}$ directions, respectively, by one pixel.

\subsection{Calculation of the matched filter}

Ideally, calculation of the matched filter would be an ongoing process, continually computed by dithering of the LGS spots on the WFS, using a tip-tilt mirror (Gilles \& Ellerbroek 2006), to allow ongoing calculation of the image gradients, $G_{x}$ and $G_{y}$. Dithering would have little effect on the AO correction since global LGS motion is ignored, being removed from the slope measurements before being passed to the reconstructor. Continual calculation means that the matched filter remains relevant over long periods while atmospheric conditions and sodium layer profile change. The introduction of a small, known, time-varying astigmatism will enable continual calculation of the relative pixel scales of both the LGS and NGS WFSs (key to maintaining AO performance in varying seeing conditions). If the introduced astigmatism is kept small (and detected using a phase locked loop), effect on image point spread function will be minimal.

However, since CANARY is an AO demonstrator, and does not need to produce astronomical science, and therefore does not require AO stability for long integration periods, we have taken the approach of building the matched filter offline to simplify on-sky operation, which is used for all results presented here. We hope to develop an online calculation capability for future sky tests and operation.

Our sequence of operations to build the matched filter is as follows.

(i) The LGS fast steering mirror is set to dither, with four separate positions per cycle. (ii) WFS slope measurements (using a COG) are recorded and used to determine the relative phase difference between the mirror and WFS. Since this is performed on averaged images, signal levels can be high, allowing the COG estimation to be accurate.

(iii) The phase of the dithering is adjusted so that the shift in WFS spots is aligned with the detector pixels and sub-apertures.

(iv) A number of WFS images are recorded, and those corresponding to each of the four dithered mirror locations are averaged together. Typically, 5-10 s of images are recorded.

(v) The COG of these four averaged images is computed, to give the dither radius (in pixels).

(vi) The image gradient, $G_{x}$ and $G_{y}$ are computed from the difference of opposing pairs of images divided by the diameter of the dither (in pixels).

(vii) The dithering is removed, and typically $5-10 \mathrm{~s}$ of images are averaged together to obtain $I_{0} . I_{1}, I_{2}, I_{3}$ and $I_{4}$ are also computed.

(viii) The matched filter, $R$, is then computed.

When computing the matched filter, we find that tuning the WFS noise level estimate, $\sigma$, can be used to improve performance, since this parameter acts to regularize part of the solution for $R$. We use a single value for $\sigma$ rather than a separate one for each pixel, partly for simplicity, and also because for a CCD detector, this is typically constant. However, we note that for a scientific CMOS detector (or indeed a multiport CCD detector) there may be performance improvements if a different value for each pixel can be used, i.e. less weight can be applied to noisier pixels.

Once the matched filter has been computed, it is uploaded to the real-time control system and matched filtering switched on. It is then necessary for us to measure reference slopes, which we do by averaging the computed wavefront slope measurements over a sufficient number of frames for wavefront turbulence to average out. Typically, we record for about $60 \mathrm{~s}$. We note however that this method of removing static wavefront offsets is impractical for larger telescope apertures, since the time to average turbulence greatly increases (Gordon, Buscher \& Baron 2011).

\subsection{Application of the matched filter}

The CANARY instrument uses a central processing unit (CPU)based real-time control system, the Durham AO real-time controller (DARC; Basden et al. 2010), which provides the necessary flexibility to implement, develop and test new algorithms. Within the real-time control system, the matched filter is applied as follows.

(i) Sub-aperture flux is computed in units of analogue to digital units (not photons), i.e. the calibrated pixels are summed.

(ii) The dot product of $R_{x}$ with the sub-aperture pixels (flattened into a vector) is computed, and the result divided by the sub-aperture flux to give the $x$ slope estimate.

(iii) Similarly for the $y$ slope estimate using $R_{y}$.

DARC has the ability to actively track Shack-Hartmann spots, i.e. the sub-aperture positions can follow the spots, such that the spots remain close to the centre of the sub-aperture, based on the location of the spot in the previous image frame. This therefore aids the linearity of the matched filter algorithm, since the matched filter will be applied to spots that are close to the sub-aperture centre. The alternative to this approach, which we do not explore, is a twostep algorithm, which initially estimates spot position with a COG algorithm, and then applies the matched filter to this. However, this introduces additional computational complexity. 


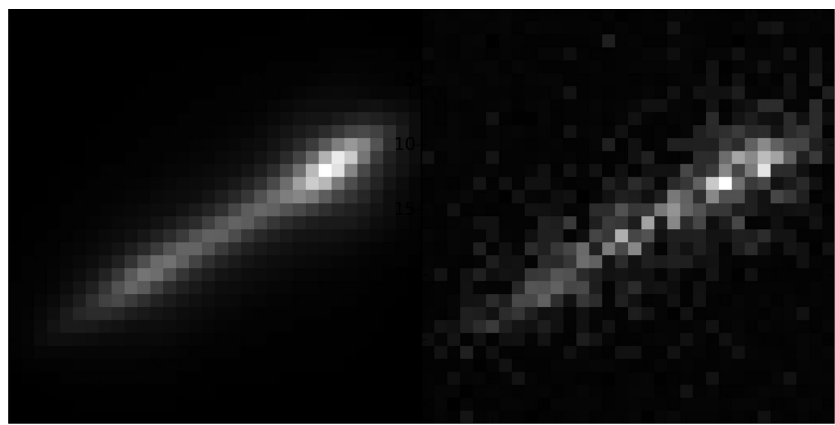

Figure 2. An example atmospherically propagated sodium LGS ShackHartmann spot, as used in simulations (6.4 arcsec field of view). Left: noiseless image. Right: with added photon, show noise (for a total flux of 1000 photons) and readout noise (0.1 electrons per pixel RMS).

Within DARC, we use a different matched filter for each subaperture, and internally, these are stored in a two-dimensional array where the location of the matched filter corresponds with the default sub-aperture position. Therefore, we can apply matched filtering to sub-apertures of different sizes, and to different spot elongations across the pupil.

\subsection{Development and testing of matched filters}

To develop the real-time implementation of the matched filter algorithm and to test the technique used to build the matched filter, we employed two simulation concepts. First, we use Durham AO simulation platform (DASP; Basden et al. 2007) to model a single sub-aperture with an extended LGS spot viewed through atmospheric turbulence. We use this simulation tool to build the matched filter as outlined in Section 2. We then generate a sequence of atmospherically propagated Shack-Hartmann spots, as shown in Fig. 2 and compare the COG and matched filter spot position estimators with the true known location (measured on a noiseless image using a COG). The RMS deviation of each estimator from the known position is then computed. We are able to explore matched filter performance covering a parameter space including elongation, signal level, readout noise level and number of frames used when building the matched filter. Although we do not seek to present significant simulated results here, because matched filter performance has been studied elsewhere, for example, Conan et al. (2009), we present a summary of our modelling. This modelling is based on an LGS launched $40 \mathrm{~m}$ from the telescope aperture (approximating CANARY and furthest sub-apertures of the E-ELT), and a $60 \mathrm{~cm}$ sub-aperture. We note that for the TMT, which uses centre-launched LGSs, maximum sub-aperture distance from the launch axis will be about $15 \mathrm{~m}$. Default photon flux in our simulations is 1000 photons per sub-aperture per frame (pessimistic compared with on-sky measurements by a factor of about 2-5; Bonaccini Calia 2016) with sub-electron readout noise (i.e. an EMCCD). Seeing of 1 arcsec is assumed, and by default we use 1000 images to build the matched filters.

After verification of the matched filter algorithm, we proceeded to develop the real-time implementation and support tools. To verify the operation of these, we use the real-time simulation concept (Basden 2014) coupling a Monte Carlo simulation tool (DASP) to the real-time control system used by CANARY. This concept is simple: instead of using real physical cameras and DMs, they are instead simulated, with the simulation WFS camera data being fed into the real-time control system. The DM command output is
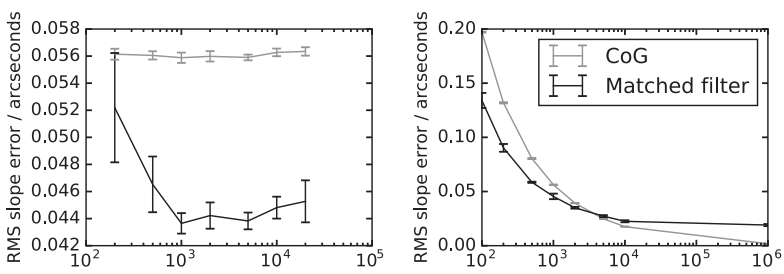

Number of samples used for matched filter

Sub-aperture flux / photons per frame
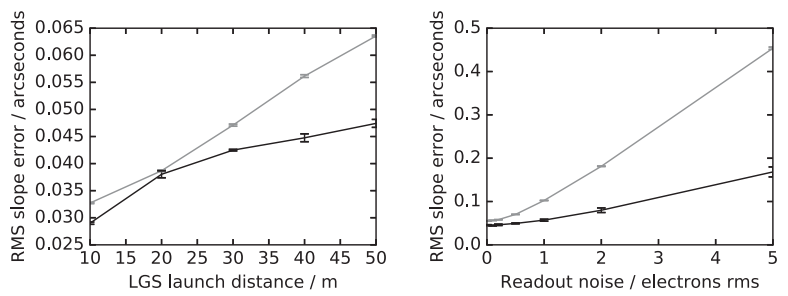

Figure 3. A figure comparing matched filtering with COG. The sub-plots give performance as RMS estimation slope error (in arcseconds) with respect to a noiseless $\mathrm{CoG}$ measurement (lower error is better performance). These plots show performance (a) as a function of number of images used to build the matched filter, (b) as a function of guide star flux, (c) as a function of distance of the LGS from the telescope axis and (d) as a function of detector readout noise.

captured and used to shape simulated DMs. We are then able to use the user interface tools developed for on-sky operation with this simulated system, and therefore provide a thorough testing, significantly reducing necessary on-sky commissioning time. The real-time control system itself does not need to know whether it is operating with real or simulated cameras and DMs.

We note that since the CANARY real-time control system is CPU based, we do not need to operate the real-time simulation facility on the same computational hardware, i.e. we can, for example, operate the simulation and real-time control system on a developer's PC, to aid development when not present at the telescope, and to allow multiple instances of the system to be run simultaneously.

By using this real-time simulation, we are then able to test building of the matched filter (including dithering), and application of the matched filter, including engaging the AO loop.

\section{MATCHED FILTERING RESULTS}

We performed tests of matched filtering using the CANARY AO onsky demonstrator instrument over a period of three nights in 2016 September. During this operation, the WLGSU launch telescope was set about $40 \mathrm{~m}$ from the WHT to generate the sodium LGS in the mesosphere. Therefore, spots were highly elongated, as shown in Fig. 1.

\subsection{Simulation measurements}

Exploration of the matched filter parameter space was used to confirm the range of expected parameters over which matched filtering would outperform a COG algorithm. Fig. 3 shows some of these results that we obtained with simulation, and it can be seen (in agreement with previous studies) that matched filtering can yield improved performance when compared with COG. We note that at high light levels, the COG performs better since we are using a noiseless COG measurement as the 'truth'.

During AO operation, if the matched filter is to be updated, reference slopes will also require updating. Within simulation, we developed a technique to compute updated reference slopes. 
(i) Compute COG and matched filter measurements of the reference image, $I_{0}$, giving $c_{x}, c_{y}, m_{x}$ and $m_{y}$, respectively, defined in pixels offset for the $x$ and $y$ directions.

(ii) Add the matched filter estimates, $m_{x}$ and $m_{y}$, to the existing reference slopes.

(iii) Subtract the COG estimates, $c_{x}$ and $c_{y}$, from the existing reference slopes.

If a matched filter is already in use, i.e. the matched filter is to be updated, an additional step is required, equal to the reverse of the above, i.e. adding the $\mathrm{COG}$ of the original image and subtracting the matched filter estimate of the original image. We note that a similar approach has been successfully taken for correlation wavefront sensing (Basden et al. 2014).

\subsection{On-bench testing}

We used the CANARY laboratory sky simulator (which uses LED light sources and two rotating phase screens to mimic the sky) to confirm that the matched filtering tools were operating as expected, dithering the sources, and building the matched filters. The WFS noise (due to shot noise and readout noise) was seen to be reduced by more than a factor of 4 (from $400 \mathrm{~nm}$ to $90 \mathrm{~nm}$ ), reducing total wavefront error (in the first 36 Zernike modes) from $950 \mathrm{~nm}$ to $750 \mathrm{~nm}$ RMS, for the configuration in which it was used, confirming that the matched filter was working. To compute WFS noise, we compute noise variance of the wavefront slope measurements, and propagate this through a theoretical Zernike reconstruction matrix, and sum the first 36 terms, as is done by Vidal et al. (2014).

\subsection{On-sky measurements}

In this section, the on-sky performance is given in term of Strehl ratio as directly measured in the $H$-band infrared images recorded by the on-axis CANARY science camera. The AO loop is always closed on the on-axis elongated LGS for the high-order correction while the tip and tilt correction is provided by the CANARY onaxis truth WFS. The LGS slope estimation algorithm was alternated between COG and matched filtering.

The COG algorithm used as the reference for these performance comparisons was well optimized: we use the brightest pixel selection algorithm (Basden, Myers \& Gendron 2012) to significantly reduce the effect of detector readout noise, with about 90 brightest pixels being selected, this number having been chosen for best performance, as shown in Fig. 4. In this case, the 90 brightest pixels are used for calculation of the COG, after a threshold equal to the value of the 91st pixel is applied. Reference slopes for both the COG and matched filter were measured on-sky, averaging 10000 frames of data (66 s) before using each algorithm.

\subsubsection{September 16}

Initial on-sky measurements were taken on the night beginning on 2016 September 16. The LGS was elongated to about 13 arcsec as measured by the WFS, and the elongation was aligned with the horizontal axis of the WFS. The amplitude of the LGS dither was set to 0.1 arcsec diameter, and 1000 frames of data were used (250 at each dither location) during recording of the image gradient, $G$, and a separate 1000 frames used to measure $I_{0}$ (without dithering). The frame rate was $150 \mathrm{~Hz}$, so $1.6 \mathrm{~s}$ of data were recorded for each dither location.

These results were measured close to dawn, when sky background was increasing. Eventually, the NGS tip-tilt signal was insufficient

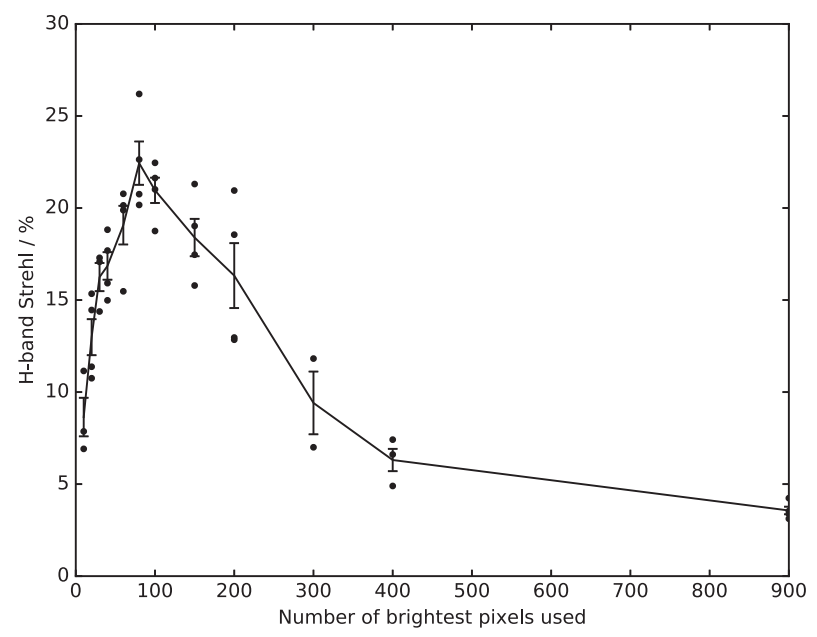

Figure 4. A figure showing AO performance as a function of number of brightest pixels chosen for the LGS WFS when using a COG algorithm. Closed-loop $H$-band Strehl ratio is shown, with the LGS used for high-order correction, and an on-axis NGS for tip-tilt correction. The line with error bars is the standard deviation computed from the available information (shown) for each number of pixels. The LGS had about $13 \mathrm{arcsec}$ of elongation.

to provide good correction, so we did not record further data. We interleaved COG and matched filter measurements.

A single matched filter was used for the duration of the experiment on this night. The matched filter computed from the data is shown in Fig. 5, along with the components used to make it, $I_{0}, G_{x}$ and $G_{y}$. We note that a few streaks are seen in these images due to problems with detector voltage levels (currently being repaired).

Fig. 6 shows measured AO system performance with and without matched filtering. It should be noted that due to atmospheric variability, we interleave measurements, so that trends in seeing with time can be somewhat mitigated. We present these results as a function of $r_{0}$, which is computed from the average value computed using the pseudo-open-loop slope measurements from the three offaxis NGS WFSs using standard CANARY tools (Vidal et al. 2014). It is evident that the matched filter is providing significantly better performance than the COG algorithm.

\subsubsection{September 17}

Further investigation of matched filtering performance was carried out, with the LGS spots elongated to about 13 arcsec, at an angle of about $10 \mathrm{deg}$ from the horizontal axis of the WFS. This time, the matched filter was built using a reference image $\left(I_{0}\right)$ that was computed while dithering, using a shift-and-add of the Shack-Hartmann images, based on the COG of the spots.

Strehl ratio was recorded using the CANARY infrared camera, at the $H$ band, and Fig. 7 shows these results. There is significant scatter due to variable seeing conditions. However, matched filtering can be seen to deliver performance improvements compared to COG, increasing the mean Strehl ratio by a factor of greater than 1.1.

\subsubsection{September 19}

When building a matched filter, the dither amplitude used will affect performance. We therefore investigated three different dither amplitudes, setting it first to 0.2 arcsec diameter, then to 0.1 arcsec (as on previous nights) and finally to 0.05 arcsec. Again, the LGS spots were elongated to about 13 arcsec at an angle of about $10 \mathrm{deg}$. In this case, the reference image, $I_{0}$, was obtained from averaging 


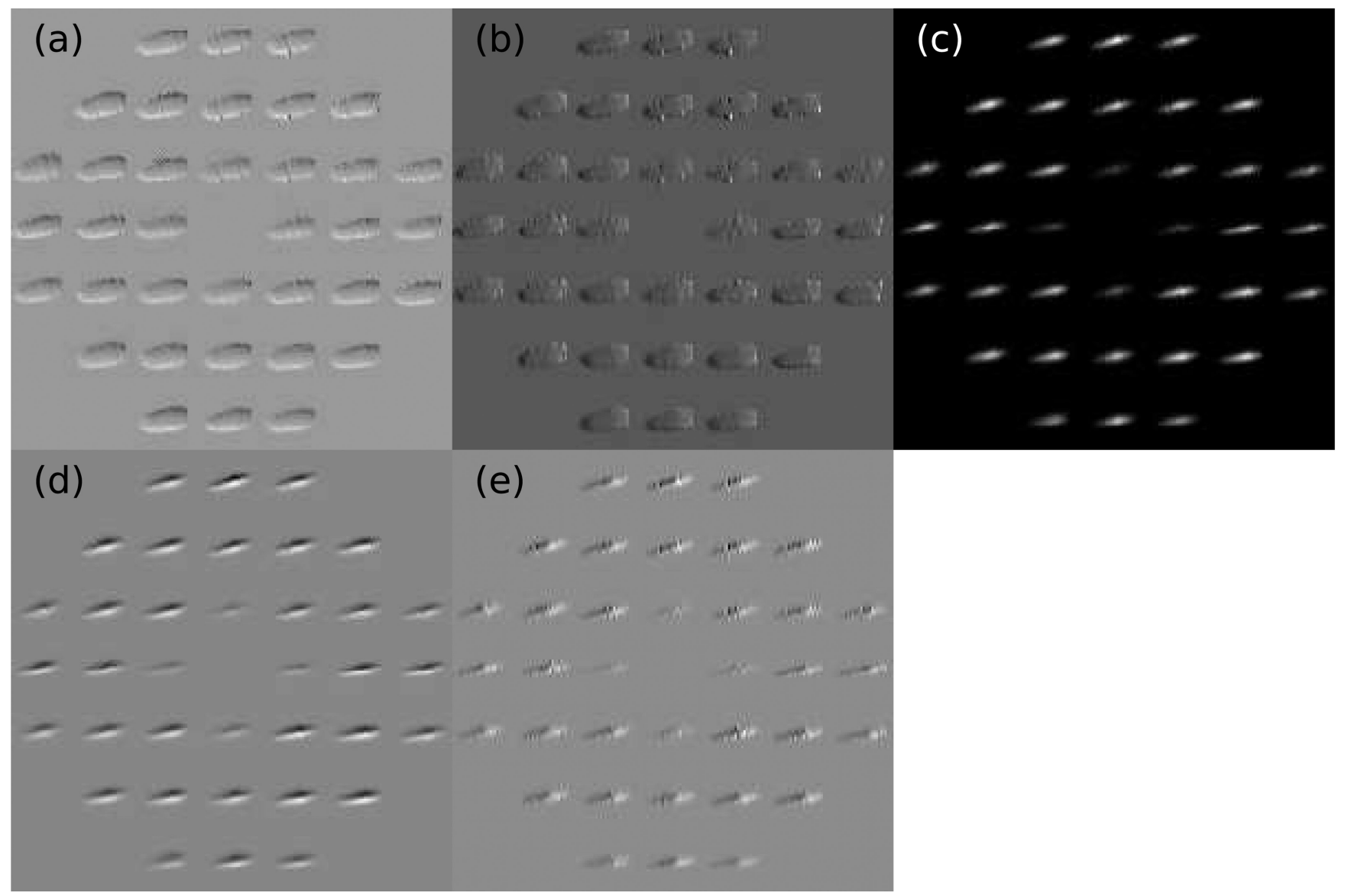

Figure 5. A figure showing, for the $7 \times 7$ sub-apertures of the CANARY phase D LGS, (a) an on-sky matched filter $x$ component, (b) the matched filter $y$ component, (c) $I_{0}$, (d) $G_{x}$ and (e) $G_{y}$. We note that we had some trouble with detector voltage levels, resulting in a few streaks in the image. This is manifested within the matched filter.

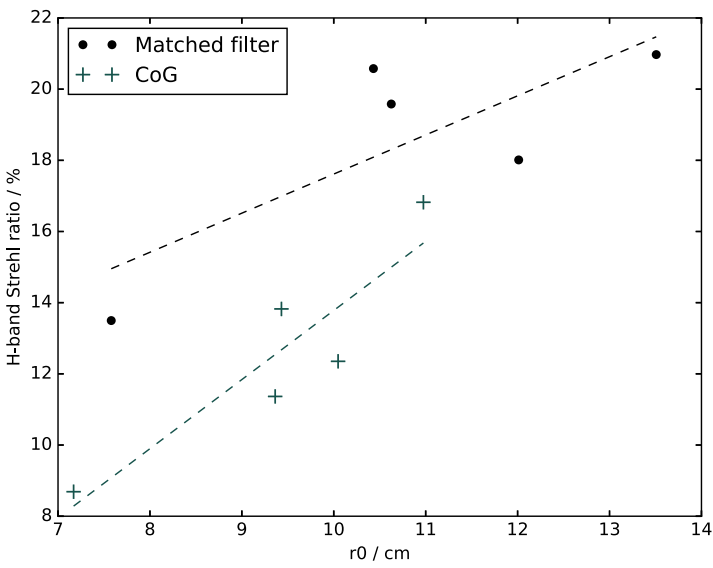

Figure 6. A figure showing $H$-band Strehl ratio for $\mathrm{CoG}$ and matched filtering measured on the night of September 16. Data are plotted as a function of Fried's parameter, $r_{0}$, which is related to seeing. A $2 \mathrm{~s}$ exposure time is used for the science images, for a star with a $V$-band apparent magnitude of 9.47. The LGS WFS is recording approximately 3000 photons per sub-aperture per frame. The matched filter and corresponding reference slopes were recorded preceding acquisition of the data.

1000 image frames without dithering (and without shift-and-add). As before, reference slopes were measured on-sky before the AO loop was closed for each matched filter and COG acquisition, in this case, with each dither amplitude.

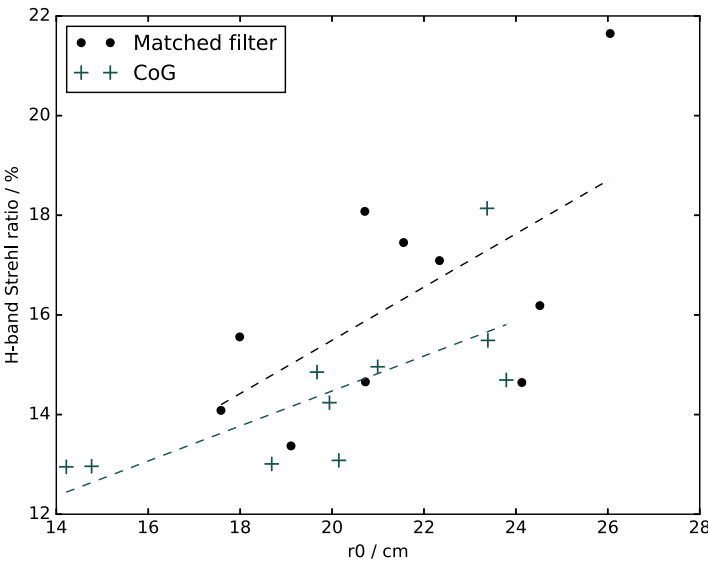

Figure 7. A figure showing $H$-band Strehl ratio as a function of Fried's parameter for the different centroiding modes measured on the night of September 17. Black circles represent matched filtering, while crosses are for COG. Mean Strehl values are given in the legend. The same target was used as on the previous night.

Fig. 8 shows the AO performance obtained. Again, it can be seen that the matched filter algorithm gives consistently better performance than the COG, except for the case with a dither amplitude of 0.05 arcsec, where the performance gain is marginal. The COG and matched filter measurements are given as a function of Fried's 

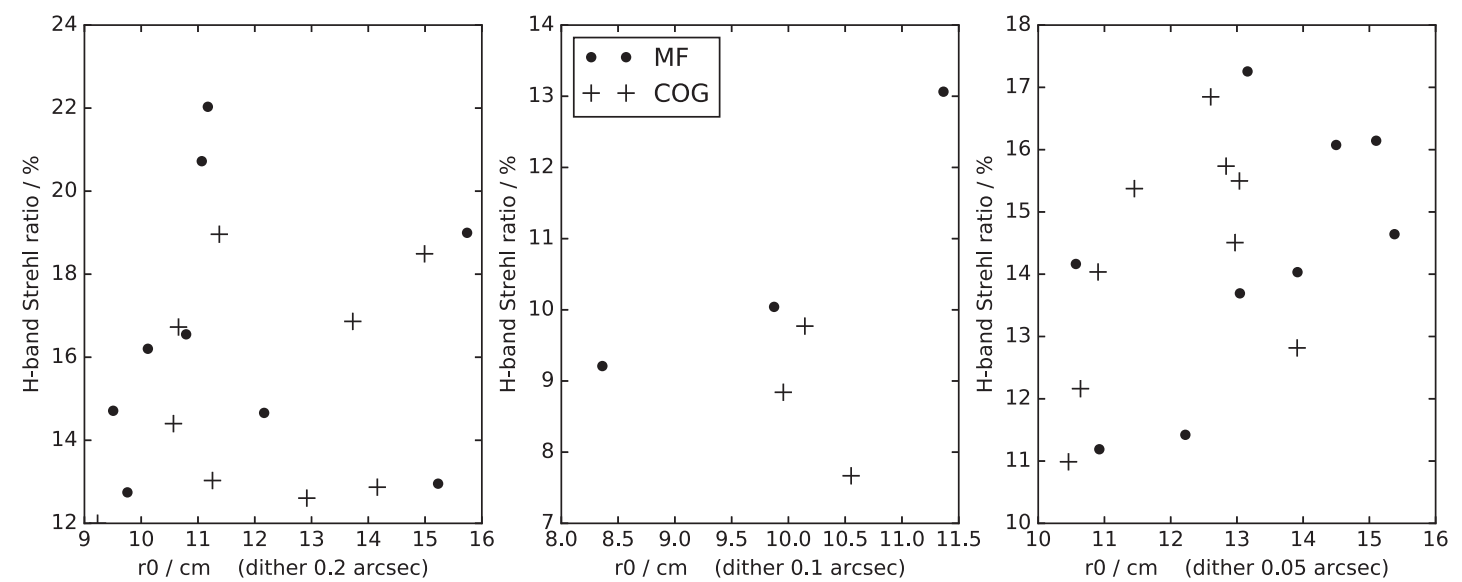

Figure 8. A figure showing AO performance ( $H$-band Strehl) for different closed-loop data sets measured on the night of September 19 using matched filtering and COG algorithms, as a function of Fried's parameter. The mean of these measurements are given in Table 1. In this case, the science target has an apparent magnitude of 8.98 and a $3 \mathrm{~s}$ exposure is used. The matched filter, and reference slopes for both COG and matched filter were recorded immediately before the start of each data set. The dither amplitude used to create the matched filter is, from left to right, $0.2,0.1$ and 0.05 arcsec.

Table 1. A table showing matched filter and COG performance for different dither amplitudes. The relative performance improvement when using matched filtering is also given.

\begin{tabular}{lcrl}
\hline $\begin{array}{l}\text { Dither } \\
\text { amplitude }\end{array}$ & $\begin{array}{l}\text { Matched filter } \\
\text { Strehl/\% }\end{array}$ & \multicolumn{1}{l}{$\begin{array}{l}\text { COG } \\
\text { Strehl/\% }\end{array}$} & Improvement(\%) \\
\hline 0.2 arcsec & $16.6 \pm 1.1$ & $15.1 \pm 0.9$ & 10 \\
$0.1 \operatorname{arcsec}$ & $10.8 \pm 1.2$ & $8.8 \pm 0.6$ & 23 \\
$0.05 \operatorname{arcsec}$ & $14.3 \pm 0.7$ & $14.2 \pm 0.6$ & 1 \\
\hline
\end{tabular}

parameter, $r_{0}$, so as to account for variable atmospheric seeing. We note that there is no algorithmic difference between the different CoG points in each figure; they were just recorded at different times (interleaved with the corresponding matched filter measurements), and hence different atmospheric conditions, using different sets of on-sky reference slopes. The mean matched filter measurements (Table 1) show an improvement over the corresponding COG measurements, though we note that due to differing seeing, computing a mean Strehl ratio does not give an accurate performance estimate; rather it serves to give us a rough idea of relative performance. From these measurements, and from Fig. 8(c), it is evident that a dither of 0.05 arcsec is too small for us to build a matched filter (this equates to 7 per cent of a pixel).

\subsubsection{On-sky results summary}

We have explored matched filtering performance with E-ELT scale extended LGS Shack-Hartmann images over several nights of operation, and in each case, we have found that matched filtering leads to AO performance improvements when compared with COG.

We note that in our case, because all sub-apertures were well elongated, the gain in WFS performance using the matched filter was evident. For an ELT, with elongation varying more significantly across the WFS, the total performance gain may be smaller, though still significant. We also note that the LGS elongation was varying during the night and from night to night, following the sodium layer thickness variations (Moussaoui et al. 2010).

\subsection{Open-loop considerations}

Our tests of matched filtering have been implemented using a closed-loop AO system, and so measured performance may be less affected by non-linearity effects than would be seen using an open-loop AO system, since the AO loop helps to maintain the Shack-Hartmann spots close to their reference positions. Therefore, performance with an open-loop AO system may not be so positive since spot motion will be larger, and spots may travel outside the linear range of the matched filter. In this case, either a two-step algorithm is required (using an initial COG estimate to find the approximate location to apply the matched filter to), or the use of adaptive sub-aperture windowing, allowing the sub-aperture to track the spot motion.

There are no proposed ELT instruments that are entirely open loop: although the proposed MOSAIC instrument (Hammer et al. 2014) on the E-ELT has an open-loop multiobject AO system, it operates with a closed-loop GLAO correction, and therefore, a significant amount of spot motion will be mitigated (for likely atmospheric turbulence profiles; Osborn et al. 2010; Sarazin et al. 2013). Therefore, matched filtering may still be appropriate here.

\subsection{Future work}

Although we have demonstrated LGS AO operation of a matched filter algorithm on-sky, there remains further much work to do, which we will attempt during future CANARY on-sky observing runs. Building the matched filter during AO loop operation, and determination of pixel scale change, is essential to improve $\mathrm{AO}$ observational efficiency.

Additionally, successful implementation of online reference slope calculation is also necessary: for the measurements presented here, we compute reference slopes for the matched filter algorithm on-sky, by averaging many frames of slope measurements (typically $10000)$ to average atmospheric turbulence. These slopes are then added to the reference slopes required to correct non-common path aberrations. This method does not represent efficient use of telescope time unless slope measurements are monitored and updated continuously while in operation. Therefore, a method to determine new matched filter reference slopes using the matched filter and 
initial reference slopes (either from a COG or previous matched filter) is required.

A further in-depth study of matched filter performance for different LGS sodium layer profiles and elongations is also necessary, with the improvements in wavefront error being calculated when the matched filter algorithm is used. Moreover, we will compare the matched filtering algorithm with other slope estimation methods in greater depth. The comparison with other slope estimation methods requires a sufficient number of on-sky observations to be statistically meaningful, hence more observing time.

\section{CONCLUSIONS}

We have successfully implemented and demonstrated a noiseoptimal matched filtering algorithm for determination of local wavefront gradients using a Shack-Hartmann sensor. On-sky testing was performed using the CANARY AO demonstrator instrument on the WHT together with the ESO Wendelstein LGS unit.

We find that matched filtering can deliver AO performance improvements compared with the conventional COG algorithm, and we measure improvements in $H$-band Strehl ratio of up to about 10 per cent (i.e. the Strehl improves by a factor of 1.1).

We therefore conclude that a matched filtering algorithm is well suited for AO systems with elongated Shack-Hartmann spot patterns, providing better performance than the commonly used COG algorithm. Our demonstration represents a significant risk mitigation though further work is required. Direct comparisons with other methods, such as correlation, also have to be tested.

\section{ACKNOWLEDGEMENTS}

This work is funded by the UK Science and Technology Facilities Council, grant ST/K003569/1, and a consolidated grant ST/L00075X/1. The ESO participation and installed infrastructure is funded under the ESO Technology Development Program. This work was partially supported by the European Commission (Fp7 Infrastructures 2012-1, OPTICON Grant 312430, WP1). This work is also supported by the European Southern Observatory. CANARY is a visitor instrument to the William Herschel Telescope, operated by the Isaac Newton Group in the Spanish Observatorio del Roque de los Muchachos of the Instituto de Astrofisica de Canarias.

\section{REFERENCES}

Basden A. G., 2014, MNRAS, 439, 2854

Basden A. G., Butterley T., Myers R. M., Wilson R. W., 2007, Appl. Opt., 46, 1089

Basden A. G., Geng D., Myers R., Younger E., 2010, Appl. Opt., 49, 6354

Basden A. G., Myers R. M., Gendron E., 2012, MNRAS, 419, 1628

Basden A. G., Chemla F., Dipper N., Gendron E., Henry D., Morris T., Rousset G., Vidal F., 2014, MNRAS, 439, 968

Basden A. G., Morris T. J., Gratadour D., Gendron E., 2015, MNRAS, 449, 3537

Basden A. G. et al., 2016, MNRAS, 459, 1350
Bonaccini Calia D., 2016, in Marchetti E., Close L. M., Véran J.-P., eds, Proc. SPIE Conf. Ser. Vol. 9909, Adaptive Optics Systems V. SPIE, Bellingham, p. 99095E

Bonaccini Calia D. et al., 2012, in Navarro R., Cunningham C. R., Prieto E., eds, Proc. SPIE Conf. Ser. Vol. 8450, Modern Technologies in Spaceand Ground-based Telescopes and Instrumentation II. SPIE, Bellingham, p. $84501 \mathrm{R}$

Conan R., Lardière O., Herriot G., Bradley C., Jackson K., 2009, Appl. Opt., 48,1198

Foy R., Labeyrie A., 1985, A\&A, 152, L29

Fusco T. et al., 2006, Opt. Express, 14, 7515

Gach J.-L. et al., 2016, in Gavel D., Trancho G., eds, OCAM2S: An Integral Shutter Ultrafast and Low Noise Wavefront Sensor Camera for Laser Guide Stars Adaptive Optics Systems. Univ. of California, California, p. 58

Gendron E., Vidal F., Brangier M., Morris T., Hubert Z., Basden A. G., Rousset G., Myers R., 2011, A\&A, 529, L2

Gilles L., Ellerbroek B., 2006, Appl. Opt., 45, 6568

Gilles L., Ellerbroek B. L., 2008, Opt. Lett., 33, 1159

Gordon J. A., Buscher D. F., Baron F., 2011, Appl. Opt., 50, 5303

Hammer F. et al., 2014, in Ramsay S. K., McLea I. S., Takami H., eds, Proc. SPIE Conf. Ser. Vol. 9147, Ground-based and Airborne Instrumentation for Astronomy V. SPIE, Bellingham, p. 914727

Jovanovic N. et al., 2015, PASP, 127, 890

Macintosh B. A. et al., 2012, in McLean I. S., Ramsay S. K., Takami H., eds, Proc. SPIE Conf. Ser. Vol. 8446, Ground-based and Airborne Instrumentation for Astronomy IV. SPIE, Bellingham, p. 84461U

Moussaoui N., Clemesha B. R., Holzlöhner R., Simonich D. M., Bonaccini Calia D., Hackenberg W., Batista P. P., 2010, A\&A, 511, A31

Myers R. M. et al., 2008, in Hubin N., Max C. E., Wizinowich P. L., eds, Proc. SPIE Conf. Ser. Vol. 7015, Adaptive Optics Systems. SPIE, Bellingham, p. $70150 \mathrm{E}$

Nelson J., Sanders G. H., 2008, in Stepp L. M., Gilmozzi R., eds, Proc. SPIE Conf. Ser. Vol. 7012, Ground-based and Airborne Telescopes II. SPIE, Bellingham, p. 70121A

Osborn J., Wilson R., Butterley T., Shepherd H., Sarazin M., 2010, MNRAS, 406, 1405

Rigaut F. et al., 2012, in Ellerbroek B. L., Marchetti E., Véran J.-P., eds, Proc. SPIE Conf. Ser. Vol. 8447, Adaptive Optics Systems III. SPIE, Bellingham, p. 84470I

Rousset G. et al., 2014, in Marchetti E., Close L. M., Véran J.-P., eds, Proc. SPIE Conf. Ser. Vol. 9148, Adaptive Optics Systems IV. SPIE, Bellingham, p. 91483M

Sarazin M., Le Louarn M., Ascenso J., Lombardi G., Navarrete J., 2013, in Esposito S., Fini L., eds, Adaptative Optics for Extremely Large Telescopes 3. Arcetri, Italy

Tamai R., Spyromilio J., 2014 in Stepp L. M., Gilmozzi R., Hall H. J., eds, Proc. SPIE Conf. Ser. Vol. 9145, Ground-based and Airborne Telescopes V. SPIE, Bellingham, p. 91451E

Thomas S., Fusco T., Tokovinin A., Nicolle M., Michau V., Rousset G., 2006, MNRAS, 371, 323

Vidal F. et al., 2014, A\&A, 569, A16

This paper has been typeset from a $\mathrm{T}_{\mathrm{E}} \mathrm{X} / \mathrm{L} \mathrm{T}_{\mathrm{E}} \mathrm{X}$ file prepared by the author. 